\title{
Adaptive State Tracking Controller for Multi-Input Multi-Output Non-affine Nonlinear Systems
}

\author{
Reza Ghasemi
}

\begin{abstract}
This paper proposes a new method to design a fuzzy adaptive controller for a class of multi-input multi-output (MIMO) non-affine nonlinear systems in which functions of the systems are unknown. The lyapunov stability of the closed loop system is guaranteed. The proposed controller is robust against uncertainty and external disturbance. An illustrative example shows the effectiveness of the proposed method and the simulation results are very promising.
\end{abstract}

Index Terms-Fuzzy Adaptive Controller- Nonlinear System- closed loop stability- MIMO Systems.

\section{INTRODUCTION}

Nowadays, fuzzy adaptive controller (FAC) attracts many researchers to developed appropriate controllers for nonlinear systems because of the following reasons.

Due to its tunable structure, the performance of the FAC is superior that of the fuzzy controller.

Instead of using adaptive controller, FAC can use knowledge of the experts in the controller.

In the recent year, FAC has been fully studied as follow:

1. The TS fuzzy systems have been used to model nonlinear systems and then TS based controllers have been designed with guaranteed stability $[16,15]$. To model affine nonlinear system and to design stable TS based controllers have been employed in [17]. Designing of the sliding mode fuzzy adaptive controller for a class of multivariable TS fuzzy systems are presents in [20]. In [18, 19], the non-affine nonlinear function are first approximated by the TS fuzzy systems, and then stable TS fuzzy controller and observer are designed for the obtained model. In these papers, modeling and controller has been designed simply, but the systems must be linearizable around some operating points.

2. The linguistic fuzzy systems have been used to design controllers for nonlinear systems.

$[1,25,22]$ have considered linguistic fuzzy systems to design stable adaptive controller for affine systems based on feedback linearization and furthermore in [22], it has considered that the zero dynamic is stable. Stable FAC based on sliding mode is designed for affine systems in [26]. Designing of the FAC for affine chaotic systems are presented in [23, 24]. To design stable FAC and linear observer for class of affine nonlinear systems are presented in $[12,11,14,13]$. Fuzzy adaptive sliding mode controller is presented for class of affine nonlinear time delay systems in $[2,21,6]$. The output feedback FAC for class of affine

Manuscript received October 10, 2010; revised May 8, 2011.

Reza Ghasemi is with the Department of Electrical Engineering, Islamic Azad University, Damavand Branch, Damavand, Iran (e-mail: rezaghasemi@damavandiau.ac.ir ). nonlinear MIMO systems is suggested in [4]. [10] designed FAC for a class of affine nonlinear time delayed systems. The main incompetency of these papers is those restricted conditions.

$[27,28]$ are involved stable FAC for class of non-affine nonlinear systems. The inefficiency of these papers is bad performance of the controller when the controller has not been adjusted. [29, 30] proposed a decentralized fuzzy model reference state tracking controller for a class of canonical nonlinear large scale system. The main limitations of these references are both considering the interaction as a bounded disturbance and availability of all states.

In this paper, we propose a new method to design an adaptive controller based on fuzzy systems for a class of non-affine nonlinear systems with guaranteed stability. The controller is robust against uncertainties, external disturbances and approximation errors.

The rest of the paper is organized as follows. Section 2 gives problem statement. General concept of the fuzzy systems is formulated in section 3. To design Fuzzy adaptive controller is proposed in section 4. Section 5 shows simulation results of the proposed controller and Section 5 concludes the paper.

\section{PROBlem STATEMENT}

The following non-affine nonlinear system has been considered in this paper.

$$
\left\{\begin{array}{l}
\underline{\dot{x}}=A \underline{x}+F(\underline{x}, \underline{u})+d(t) \\
y=C^{T} \underline{x}
\end{array}\right.
$$

where $\mathrm{X}=\left[\mathrm{x}_{1}, \ldots, \mathrm{x}_{\mathrm{n}}\right]^{\mathrm{T}} \in \mathbb{R}^{\mathrm{n}}$ is the state vector of the system which is assumed available for measurement, $\mathrm{u}=\left[\begin{array}{llll}u_{1} & u_{2} & \cdots & u_{n}\end{array}\right]^{T} \in \mathbb{R}^{\mathrm{n}}$ is the control input, $\mathrm{y} \in \mathbb{R}^{\mathrm{m}} \quad$ is the system output, $\mathrm{F}(\underline{\mathrm{x}}, \mathrm{u})=\left[\begin{array}{lll}\mathrm{f}_{1}\left(\underline{\mathrm{x}}, \mathrm{u}_{1}\right) & \cdots & \mathrm{f}_{\mathrm{n}}\left(\underline{\mathrm{x}}, \mathrm{u}_{\mathrm{n}}\right)\end{array}\right]^{\mathrm{T}}$ are an unknown smooth nonlinear function, and $d(t)$ is bounded disturbance.

The control objective is to design an adaptive fuzzy controller for system (1) such that the system output $\mathrm{y}(\mathrm{t})$ follows a desired trajectory $x_{\mathrm{m}}(\mathrm{t})$ while all signals in the closed-loop system remain bounded.

In this paper, we will make the following assumptions concerning the system (1) and the desired trajectory $x_{\mathrm{m}}(\mathrm{t})$.

Assumption 1: without loss of generality, it is assumed that the nonzero function $\mathrm{f}_{\mathrm{u}}(\mathrm{x}, \mathrm{u})=\partial \mathrm{f}(\mathrm{x}, \mathrm{u}) / \partial \mathrm{u}$ satisfies the 
following condition:

$$
\begin{gathered}
\mathrm{f}_{\mathrm{iu}}\left(\underline{\mathrm{x}}, \mathrm{u}_{\mathrm{i}}\right) \triangleq \frac{\partial \mathrm{f}_{\mathrm{i}}\left(\underline{\mathrm{x}}, \mathrm{u}_{\mathrm{i}}\right)}{\partial u_{i}} \geq \mathrm{f}_{\mathrm{imin}}>0 \\
\forall(\underline{\mathrm{x}}, \mathrm{u}) \in \mathbb{R}^{\mathrm{n}} ? \mathbb{R}, 1 \leq i \leq n \\
\frac{d \mathrm{f}_{\mathrm{iu}}(\underline{\mathrm{x}}, \mathrm{u})}{d t} \geq \mathrm{f}_{\mathrm{idm}} \\
\mathrm{f}_{\mathrm{imin}}, \mathrm{f}_{\mathrm{idm}} \in \mathbb{R} \text { are known and constant. }
\end{gathered}
$$

Assumption 2: The desired trajectory $x_{\mathrm{m}}(\mathrm{t})$ is generated by the following equation.

$$
\left\{\begin{array}{l}
\underline{x}_{m}=A \underline{x}_{m}+B \underline{r}(t) \\
y_{m}=C^{T} \underline{x}_{m}
\end{array}\right.
$$

$r(t)$ is the external reference signal. A and B are chosen so that the reference signal mentioned in equation (3) be stable.

Assumption 3: the disturbance in the above equation is bounded by:

$$
\|d(t)\|_{\infty} \leq d_{\max }
$$

Define the tracking error vector as:

$$
\underline{\mathrm{e}}=\underline{\mathrm{x}}_{\mathrm{m}}-\underline{\mathrm{x}}
$$

Taking the derivative of both sides of the equation (5) we have

$$
\left\{\begin{array}{l}
\underline{\dot{\mathrm{e}}}=\dot{\mathrm{x}}_{\mathrm{m}}-\underline{\dot{\mathrm{x}}}=A \underline{x}_{m}+B \underline{r}(t)-\mathrm{A} \underline{\mathrm{x}}-F(\underline{x}, \underline{u})-d(t) \\
e_{y}=C^{T} \underline{\mathrm{e}}
\end{array}\right.
$$

Use equation (5) to rewrite the above equation as:

$$
\left\{\begin{array}{l}
\underline{\mathrm{e}}=A \underline{\mathrm{e}}+B \underline{r}(t)-F(\underline{x}, \underline{u})-d(t) \\
e_{y}=C^{T} \underline{\mathrm{e}}
\end{array}\right.
$$

Let $\underline{v}=\left[\begin{array}{lll}v_{1} & \cdots & v_{n}\end{array}\right]^{T}$ be defined as

$$
\underline{\mathrm{v}}=\mathrm{B} \underline{\mathrm{r}}(\mathrm{t})+\mathrm{K}_{c} \underline{\mathrm{e}}+\underline{v}^{\prime}
$$

$K_{\mathrm{c}}$ chosen so that $\mathrm{A}_{\mathrm{c}}=\mathrm{A}-\mathrm{K}_{c}$ be Hurwitz.

By adding and subtracting the term $\mathrm{k}^{\mathrm{T}} \mathrm{e}+v^{\prime}$ from the right-hand side of equation (7), we obtain

$$
\left\{\begin{array}{l}
\underline{\dot{\mathrm{e}}}=\left(A-\mathrm{K}_{c}\right) \underline{\mathrm{e}}+\underline{\mathrm{v}}-\underline{v}^{\prime}-F(\underline{x}, \underline{u})-d(t) \\
\mathrm{e}_{\mathrm{y}}=\mathrm{C}^{\mathrm{T}} \underline{\mathrm{e}}
\end{array}\right.
$$

Using assumption (1), equation (11) and the signal $v$ which is not explicitly dependent on the control input $u$, the following inequality is satisfied:

$$
\frac{\partial\left(f_{i}\left(\underline{x}, u_{i}\right)-v_{i}\right)}{\partial u_{i}}=\frac{\partial f_{i}\left(\underline{x}, u_{i}\right)}{\partial u_{i}}>\mathrm{f}_{\text {imin }}>0
$$

Invoking the implicit function theorem, it is obvious that the nonlinear algebraic equation $f(\underline{x}, \underline{u})-\underline{v}=0$ is locally soluble for the input $\underline{u}$ for an arbitrary $(\underline{\mathrm{x}}, \underline{\mathrm{v}})$. Thus, there exists some ideal controller $\underline{\mathrm{u}}^{*}(\underline{\mathrm{x}}, \underline{\mathrm{v}})$ satisfying the following equality for a given $(\underline{\mathrm{x}}, \underline{\mathrm{v}}) \in \mathrm{R}^{\mathrm{n}}$ ? $\mathrm{R}$ :

$$
F\left(\underline{x}, \underline{u}^{*}\right)-\underline{v}=0
$$

As a result of the mean value theorem, there exists a constant $\lambda$ in the range of $0<\lambda<1$, such that the nonlinear function $\mathrm{F}(\underline{\mathrm{x}}, \underline{\mathrm{u}})$ can be expressed around $\underline{\mathrm{u}}{ }^{*}$ as:

$$
\mathrm{F}(\underline{\mathrm{x}}, \underline{\mathrm{u}})=\mathrm{F}\left(\underline{\mathrm{x}}, \underline{\mathrm{u}}^{*}\right)+\mathrm{F}_{\mathrm{u}_{\lambda}}\left(\underline{\mathrm{u}}^{-\underline{\mathrm{u}}^{*}}\right)=\mathrm{F}\left(\underline{\mathrm{x}}, \underline{\mathrm{u}}^{*}\right)+\mathrm{F}_{\mathrm{u}_{\lambda}} \underline{\mathrm{e}}_{\mathrm{u}}
$$

where $\mathrm{F}_{\mathrm{u}_{\lambda}}=\partial \mathrm{F}(\underline{\mathrm{x}}, \underline{\mathrm{u}}) /\left.\partial \underline{\mathrm{u}}\right|_{\underline{\mathrm{u}}=\underline{\mathrm{u}}_{\lambda}}$ and $\underline{\mathrm{u}}_{\lambda}=\lambda \underline{\mathrm{u}}+(1-\lambda) \underline{\mathrm{u}}^{*}$.

Substituting equation (15) into the error equation (12) and using (14), we get

$$
\left\{\begin{array}{l}
\dot{\mathrm{e}}=\underbrace{\left(A-\mathrm{K}_{c}\right)}_{A_{c}}-\mathrm{e}^{-}-\mathrm{F}_{\mathrm{u}_{\lambda}} \mathrm{e}_{\mathrm{u}}-d(t)-v^{\prime} \\
\mathrm{e}_{\mathrm{y}}=\mathrm{C}^{\mathrm{T}} \underline{\mathrm{e}}
\end{array}\right.
$$

However, the implicit function theory only guarantees the existence of the ideal controller $\underline{\mathrm{u}}^{*}(\underline{\mathrm{x}}, \underline{\mathrm{v}})$ for system (14), and does not recommend a technique for constructing solution even if the dynamics of the system are well known. In the following, a fuzzy system and classic controller will be used to obtain the unknown ideal controller.

\section{FUZZY SYSTEMS}

Figure (1) shows the basic configuration of the fuzzy systems considered in this paper. Here, we consider a multi-input, single-output fuzzy systems: $x \in U \subset R^{n} \rightarrow y \in V \subset R \quad$. Consider that a multi-output system can be separated into a group of single-output systems.

The fuzzifier performs a mapping from a crisp input vector $x=\left[x_{1}, x_{2}, \ldots, x_{n}\right]^{T}$ to a fuzzy set, where the label of the fuzzy set are such as "small", "medium", "large", etc.

The fuzzy rule base is consisted of a collection of fuzzy IF-THEN rules. Assume that there are $\mathrm{M}$ rules, and the $l^{\text {th }}$ rule is

$$
\begin{array}{r}
R^{l}(u): \text { if }\left(x_{1} \text { is } A_{1}^{l} \ldots x_{n} \text { is } A_{1}^{l}\right) \text { then }\left(y \text { is } B^{l}\right) \\
l=1,2, \ldots, M
\end{array}
$$

where $x=\left[x_{1}, x_{2}, \ldots, x_{n}\right]^{T}$ and $\mathrm{y}$ are the crisp input and output of the fuzzy system, respectively. $A_{j}^{l}$ and $B^{l}$ are fuzzy membership function in $U_{j}$ and $\mathrm{V}$, respectively.

The fuzzy inference performs a mapping from fuzzy sets in $\mathrm{U}$ to fuzzy sets in $\mathrm{V}$, based on the fuzzy IF-THEN rules in the fuzzy rule base.

The defuzzifier maps fuzzy sets in $\mathrm{V}$ to a crisp value in $\mathrm{V}$. The configuration of Figure (1) represents a general framework of fuzzy systems, because many different choices are allowed for each block in Figure 1, and various combinations of these choices will construct different fuzzy systems [8]. Here, we use the sum-product inference and the center-average defuzzifier. Therefore, the fuzzy system output can be expressed as

$$
y(x)=\frac{\sum_{l=1}^{M} y^{l} \prod_{i=1}^{n} \mu_{A_{i}^{l}}\left(x_{i}\right)}{\sum_{l=1}^{M} \prod_{i=1}^{n} \mu_{A_{i}^{l}}\left(x_{i}\right)}
$$


where $\mu_{A_{i}^{l}}\left(x_{i}\right)$ is the membership degree of the input xi to fuzzy set $A_{j}^{l}$ and $y^{l}$ is the point at which the membership function of fuzzy set $B^{l}$ achieves its maximum value.

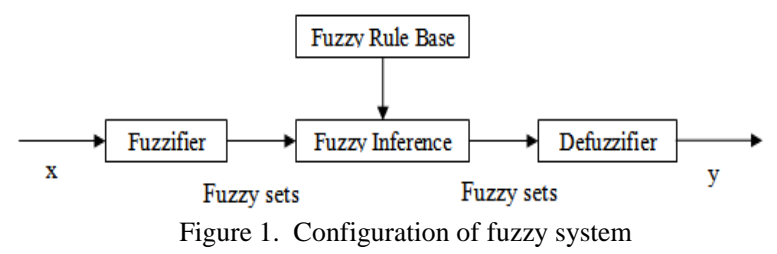

The fuzzy systems in the form of (18) are proven in [7] to be a universal approximator if their parameters are properly chosen.

Theorem 1[8]: Suppose $f(x)$ is a continuous function on a compact set $\mathrm{U}$. Then, for any $\varepsilon>0$, there exists a fuzzy system like (18) satisfying:

$$
\sup _{x \in U}|f(x)-y(x)| \leq \varepsilon
$$

The output given by (18) can be rewritten in the following compact form:

$$
y(x)=w(x)^{T} \theta
$$

where $\theta=\left[y^{1} y^{2} \ldots y^{M}\right] \quad$ is a vector grouping all consequent parameters,

and $w(x)=\left[w_{1}(x) w_{2}(x) \ldots w_{M}(x)\right]^{T}$ is a set of fuzzy basis functions defined as:

$$
w_{i}(x)=\frac{\prod_{i=1}^{n} \mu_{A_{i}^{l}}\left(x_{i}\right)}{\sum_{l=1}^{M} \prod_{i=1}^{n} \mu_{A_{i}^{l}}\left(x_{i}\right)}
$$

The fuzzy system (18) is assumed to be well defined so that $\sum_{l=1}^{M} \prod_{i=1}^{n} \mu_{A_{i}^{l}}\left(x_{i}\right) \neq 0$ for all $x \in U$.

\section{FuZZY AdAPTIVE CONTROLler DESIGN}

In Section 2, it has been shown that there exists an ideal control for achieving control objectives. In this section, we show how to develop a fuzzy system to adaptively approximate the unknown ideal controller.

The ideal controller can be represented as:

$$
\underline{u}^{*}=\Psi(\underline{z})
$$

where $\underline{z}=[\underline{x}, \underline{v}]^{T}, \Psi(\underline{z})=\theta^{* T} w(\underline{z})+\varepsilon_{u}$, and $\theta^{*}$ and $w(\underline{z})$ are consequent parameters and a set of fuzzy basis functions, respectively. $\varepsilon_{u}$ is an approximation error that satisfies $\left|\varepsilon_{u}\right| \leq \varepsilon_{\max }$ and $\varepsilon_{\max }>0$. The parameters $\theta^{*}$ are determined through the following optimization.

$$
\theta^{*}=\underset{\theta}{\arg \min }\left[\sup \left|\theta^{T} w(z)-\Psi(z)\right|\right]
$$

Denote the estimate of $\theta^{*}$ as $\theta$ and $\underline{u}_{r o b}$ as a robust controller to compensate approximation error, uncertainties, and disturbance to rewrite the controller given in (22) as:

$$
\underline{u}=\theta^{T} w(\underline{z})+\underline{u}_{r o b}
$$

In which $u_{r o b}$ is defined below.

$$
\begin{aligned}
& \underline{u}_{r o b}=S_{\text {sign }}\left(\underline{u}_{\text {com }}+F_{\min }^{-1} \underline{u}_{r}+F_{\min }^{-1} \hat{v}^{\prime}\right) \\
& F_{\text {min }}=\operatorname{Diag}\left(f_{1 \min }, f_{2 \min }, \cdots, f_{n \min }\right) \\
& S_{\text {sign }}=\operatorname{Diag}\left(\operatorname{sign}\left(P_{1} e_{1}\right), \operatorname{sign}\left(P_{2} e_{2}\right), \cdots, \operatorname{sign}\left(P_{n} e_{n}\right)\right)
\end{aligned}
$$

In the above, $\theta^{T} w(\underline{z})$ approximates the ideal controller, $\underline{u}_{c o m}$ compensates for approximation errors and uncertainties, $\underline{u}_{r}$ is designed to compensate for bounded external disturbances, and $\underline{v}^{\prime}$ is estimation of $\underline{v}^{\prime}$. Consider the following update laws.

$$
\begin{aligned}
& \underline{\dot{\theta}}=\Gamma \underline{P} \underline{e} w(\underline{z}) \\
& \underline{\dot{u}}_{r}=\gamma_{u_{r}} F_{\min }^{-1} S_{s i g n} \underline{P} \underline{e} \\
& \underline{\dot{u}}_{c o m}=\gamma_{u_{c o m}} F_{\min }^{-1} S_{s i g n} \underline{P} \underline{e} \\
& \dot{\hat{v}}^{\prime}=\gamma_{\hat{v}^{\prime}} F_{\min }^{-1} S_{\text {sign }} \underline{P} \underline{e}
\end{aligned}
$$

where $\quad \Gamma_{1}=\Gamma_{1}^{T}>0, \gamma_{u_{r}}>0, \gamma_{u_{c o m}}>0, \gamma_{\hat{v}^{\prime}}>0 \quad$ are constant parameters.

Define error vector $\tilde{\theta}=\theta-\theta^{*}$ and use (24) and (25) to rewrite the error equation (16) as:

$$
\left\{\begin{array}{l}
\underline{\mathrm{e}}=\underbrace{\left(A-\mathrm{K}_{c}\right)}_{A_{c}} \underline{\mathrm{e}}-\mathrm{F}_{\mathrm{u}_{\lambda}}\left(\tilde{\theta}^{T} w(\underline{z})+\underline{u}_{r o b}-\varepsilon_{u}\right)-d(t)-v^{\prime} \\
\mathrm{e}_{\mathrm{y}}=\mathrm{C}^{\mathrm{T}} \underline{\mathrm{e}}
\end{array}\right.
$$

In following equation, $\lambda_{\max (.)}$ and $s v d_{\max }($.$) are$ maximum eigenvalue and maximum singular value decomposition, respectively.

Lemma 1: The following inequality holds if

$$
\begin{aligned}
& \lambda_{\text {min }}\left(Q_{i}\right) \geq-f_{d \mathrm{~m}} / f_{\text {min }} \lambda_{\text {min }}\left(P_{i}\right) \text { for } i=1,2, \ldots, n \text {. } \\
& \frac{1}{2} \underline{e}^{T}\left(Q F_{u_{\lambda f}}^{-1}-P \dot{F}_{u_{\lambda f}}^{-1}\right) \underline{e} \geq 0
\end{aligned}
$$

Proof: for more information refer to [30].

Theorem 2: consider the error dynamical system given in (26) for the large scale system (1) satisfying assumption (1), the external disturbances satisfying assumption (3), and a desired trajectory satisfying assumption (2), then the controller structure given in (24), (25) with adaptation laws (27) makes the tracking error converge asymptotically to a neighborhood of origin and all signals in the closed loop system be bounded.

Proof: consider the following lyapunov function.

$V=\frac{1}{2}\left(\underline{e}^{T} P_{1} F_{u_{\lambda f}}^{-1} \underline{e}+\tilde{\theta}^{T} \Gamma_{1}^{-1} \tilde{\theta}+\underline{\tilde{u}}_{r}^{T} \gamma_{u_{r}} \underline{\tilde{u}}_{r}+\tilde{u}_{c o m}^{T} \gamma_{u_{c o m}} \tilde{u}_{c o m}+\tilde{v}^{\prime T} \gamma_{\hat{v}} \tilde{v}^{\prime}\right)$

where

$$
\tilde{\theta}_{1}=\theta_{1}-\theta_{1}^{*}
$$


$\tilde{u}_{r}=u_{r}-d_{\max } / f_{\text {min }} \quad, \quad \tilde{u}_{c o m}=u_{c o m}-\varepsilon_{\max } \quad, \quad$ and $\tilde{v}^{\prime}=\hat{v}^{\prime}-\left|v^{\prime}\right|$. The time derivative of the lyapunov function becomes.

$$
\begin{aligned}
\dot{V} & =\frac{1}{2}\left(\underline{e}^{T} P_{1} F_{u_{\lambda f}}^{-1} \underline{e}+\underline{e}^{T} P_{1} F_{u_{\lambda f}}^{-1} \underline{e}+\underline{e}^{T} P_{1} \dot{F}_{u_{\lambda f}}^{-1} \underline{e}\right) \\
& +\tilde{\theta}^{T} \Gamma_{1}^{-1} \dot{\theta}+\underline{\tilde{u}}_{r}^{T} \gamma_{u_{r}} \underline{\dot{u}}_{r}+\underline{\tilde{u}}_{c o m}^{T} \gamma_{u_{c o m}} \underline{\dot{u}}_{c o m}+\tilde{v}^{\prime T} \gamma_{\hat{v}^{\prime}} \dot{\hat{v}}^{\prime}
\end{aligned}
$$

Use (26), to rewrite above equation as:

$$
\begin{aligned}
\dot{V}= & \frac{1}{2}\left(A_{c} \underline{\mathrm{e}}-\mathrm{F}_{u_{\lambda}}\left(\tilde{\theta}_{1}^{T} w_{1}(\underline{z})+\underline{u}_{r o b}-\varepsilon_{u}\right)-d(t)-v^{\prime}\right)^{T} P_{1} F_{u_{\lambda f}}^{-1} \underline{e} \\
& +\frac{1}{2} \underline{e}^{T} P_{1} F_{u_{\lambda f}}^{-1}\left(A_{c} \underline{\mathrm{e}}-\mathrm{F}_{u_{\lambda}}\left(\tilde{\theta}_{1}^{T} w_{1}(\underline{z})+\underline{u}_{r o b}-\varepsilon_{u}\right)-d(t)-v^{\prime}\right)+\frac{1}{2} \underline{e}^{T} P_{1} \dot{F}_{u_{\lambda f}}^{-1} \underline{e} \\
& +\tilde{\theta}^{T} \Gamma_{1}^{-1} \dot{\theta}+\underline{\tilde{u}}_{r}^{T} \gamma_{u_{r}} \dot{u}_{r}+\tilde{u}_{c o m}^{T} \gamma_{u_{c o m}} \dot{u}_{c o m}+\tilde{v}^{\prime T} \gamma_{v^{\prime}}{\dot{v^{\prime}}}^{\prime}
\end{aligned}
$$

Use $b^{T} P e \tanh \left(b^{T} P e / \varepsilon\right)=\left|b^{T} P e\right|$ and (10), to rewrite (40) as follow.

$$
\begin{aligned}
\dot{V} & =\frac{1}{2} \underline{e}^{T}\left(A_{c}^{T} P+P A_{c}\right) F_{u_{\lambda f}}^{-1} \underline{e}+\frac{1}{2} \underline{e}_{1}^{T} \dot{F}_{u_{\lambda f}}^{-1} \underline{e} \\
& -\tilde{\theta}_{1}^{T} w_{1}\left(\underline{z}_{)}\right) P \underline{e}-\underline{u}_{r o b}^{T} P \underline{e}+\varepsilon_{u}^{T} P \underline{e}-d^{T} P F_{u_{\lambda f}}^{-1} \underline{e}-v^{T T} P F_{u_{\lambda f}}^{-1} \underline{e} \\
& +\tilde{\theta}^{T} \Gamma_{1}^{-1} \dot{\theta}+\underline{\tilde{u}}_{r}^{T} \gamma_{u_{r}} \underline{\dot{u}}_{r}+\underline{\tilde{u}}_{c o m}^{T} \gamma_{u_{c o m}} \underline{\dot{u}}_{c o m}+\tilde{v}^{\prime T} \gamma_{\hat{v}^{\prime} \hat{v}^{\prime}}
\end{aligned}
$$

Using assumptions (3), to rewrite (32) as follow.

$$
\begin{aligned}
\dot{V} & \leq-\frac{1}{2} \underline{e}^{T} Q F_{u_{\lambda f}}^{-1} \underline{e}+\frac{1}{2} \underline{e}^{T} P \dot{F}_{u_{\lambda f}}^{-1} \underline{e} \\
& -\tilde{\theta}_{1}^{T} w_{1}(\underline{z}) P \underline{e}-\underline{u}_{r o b}^{T} P \underline{e}+\varepsilon_{M a x}^{T} S_{s i g n} P \underline{e} \\
& +d_{\max }^{T} F_{\min }^{-1} S_{s i g n} P \underline{e}+v_{a b}^{T} F_{u_{\lambda f}}^{-1} S_{s i g n} P \underline{e} \\
& +\tilde{\theta}^{T} \Gamma_{1}^{-1} \dot{\theta}+\underline{\tilde{u}}_{r}^{T} \gamma_{u_{r}} \underline{\dot{u}}_{r}+\underline{\tilde{u}}_{c o m}^{T} \gamma_{u_{c o m}} \underline{\dot{u}}_{c o m}+\tilde{v}^{\prime T} \gamma_{\hat{v}^{\prime}}{\dot{\hat{v}^{\prime}}}^{\prime}
\end{aligned}
$$

The equation (33) can be rewritten as below.

$$
\begin{aligned}
& \dot{V} \leq-\frac{1}{2} \underline{e}^{T} Q F_{u_{\lambda f}}^{-1} \underline{e}+\frac{1}{2} \underline{e}^{T} P \dot{F}_{u_{\lambda f}}^{-1} \underline{e} \\
& -\tilde{\theta}_{1}^{T} w_{1}(\underline{z}) P \underline{e}-\underbrace{\left(\underline{u}_{c o m}-\varepsilon_{M a x}\right)^{T}}_{\tilde{u}_{c o m}} S_{s i g n} P \underline{e} \\
& -\underbrace{\left(\underline{u}_{r}-d_{\max }\right)^{T}}_{\underline{\tilde{u}}_{r}} F_{\min }^{-1} S_{\text {sign }} P \underline{e}-\underbrace{\left(\hat{v}^{\prime}-v_{a b}^{\prime}\right)^{T}}_{\underline{\underline{v}}^{\prime}} F_{\min }^{-1} S_{s i g n} P \underline{e} \\
& +\tilde{\theta}^{T} \Gamma_{1}^{-1} \dot{\theta}+\underline{\tilde{u}}_{r}^{T} \gamma_{u_{r}} \underline{\dot{u}}_{r}+\underline{\tilde{u}}_{c o m}^{T} \gamma_{u_{c o m}} \dot{u}_{c o m}+\tilde{v}^{\prime T} \gamma_{\hat{v}} \dot{\hat{v}}^{\prime}
\end{aligned}
$$

Using (27), the above inequality rewrites as:

$$
\dot{V} \leq-\frac{1}{2} \underline{e}^{T} Q F_{u_{\lambda f}}^{-1} \underline{e}+\frac{1}{2} \underline{e}^{T} P \dot{F}_{u_{\lambda f}}^{-1} \underline{e}
$$

Use the lemma 1, it is clear that $-\frac{1}{2} \underline{e}^{T} \underbrace{\left(Q F_{u_{\lambda f}}^{-1}-P \dot{F}_{u_{\lambda f}}^{-1}\right)}_{\leq N} \underline{e} \quad$ is negative definite.

Consequently, the following inequality can be derived.

$$
\dot{V} \leq-\frac{1}{2} \underline{e}^{T} N \underline{e}
$$

$\dot{V} \leq 0$ causes $F_{u_{\lambda_{f}}}, \underline{\hat{e}}, \underline{\tilde{e}}_{\rightarrow}, \underline{u}_{r}, \underline{u}_{c o m}, \underline{v}^{\prime}$ and $\theta$ be bounded and Lyapunov function is nondecreasing.
Equation (36) rewrites as follow.

$$
\int_{0}^{\infty} \underline{e}^{T} N \underline{e} \leq 2(V(0)-V(\infty))
$$

It is clear that $V(\infty)$ is bounded and positive then $\underline{e} \in L_{2}$ and furthermore based on boundedness of $\underline{e}$ it is clear that $\underline{e} \in L_{2} \bigcap L_{\infty}$.

Using Barbalat's lemma, it is guaranteed the tracking error asymptotically to the neighborhood of the origin. Furthermore, boundedness of the coefficient parameters is guaranteed. It completes the proof.

Q.E.D.

Remark 1. It is very important to select properly the controller parameters to gain a satisfactory performance. Here at this stage, number of the rules and the input membership functions are obtained by trial and error.

Remark 2. To guarantee the boundedness of the parameters in the presence of the approximation error, which is unavoidable, the proposed adaptive laws (27) is modified it by introducing a $\sigma$ - modification term as follows:

$$
\begin{aligned}
& \underline{\dot{\theta}}=\Gamma_{1} \underline{P} \underline{e} w_{1}(\underline{z})-\sigma \Gamma_{1} \theta_{1} \\
& \underline{\dot{u}}_{r}=\gamma_{u_{r}} F_{\min }^{-1} S_{s i g n} \underline{P} \underline{e}-\sigma \gamma_{u_{r}} u_{r} \\
& \underline{\dot{u}}_{c o m}=\gamma_{u_{c o m}} F_{\mathrm{min}}^{-1} S_{s i g n} \underline{P} \underline{e}-\sigma \gamma_{u_{c o m}} u_{c o m} \\
& \text { 资 }=\gamma_{v^{\prime}} F_{\min }^{-1} S_{s i g n} \underline{P} \underline{e}-\sigma \gamma_{v^{\prime}} v^{\prime}
\end{aligned}
$$

\section{Simulation Results}

In this section, we apply the proposed fuzzy model reference adaptive controller to MIMO nonaffine nonlinear systems [27] where describe in equation (39).

$$
\left\{\begin{array}{l}
\dot{x}_{1}=\sin \left(x_{1}\right)+\tanh \left(u_{1}\right)+\sin \left(x_{2}\right)+d_{1}(t) \\
\dot{x}_{2}=x_{2}^{2}+0.15 u_{2}^{3}+0.1\left(1+x_{1}^{2}\right) u_{2}+\sin \left(0.1 u_{2}\right)+d_{2}(t) \\
y=\left[\begin{array}{ll}
1 & 0 \\
2 & 1
\end{array}\right] \underline{x}
\end{array}\right.
$$

where $d_{1}(t)=0.5 \sin (200 \pi t), d_{2}(t)=0.5 \sin (100 \pi t)$ are bounded external disturbance. The control objective is to make the states of the system track the desire trajectories.

We consider the desired value of the external reference signals are $r_{1}=0.3 \sin (0.2 \pi t)+0.3 \sin (0.4 \pi t)$ and $r_{2}=0.3 \sin (0.3 \pi t)+0.3 \sin (0.6 \pi t)$.

Let $x=\left[x_{1}, x_{2}\right]^{T}, z=\left[x_{1}, x_{2}, v\right]^{T}$ and $v$ are defined over $[-5,5],[-5,5]$ and $[-40,40]$, respectively. For each fuzzy system input, we define 6 membership functions over the defined sets and furthermore all of the membership functions are defined by the Gaussian function $\mu_{j}(\chi)=\exp \left((\chi-c)^{2} / 2 \delta^{2}\right)$, where $c$ is the center of the membership function and $\delta$ is its variance. We assume that the initial value of controller parameters be zero. 
Furthermore, it has been assumed that $F_{\min }=\operatorname{Diag}(1,1) \quad, \quad \Gamma_{1}=\operatorname{Diag}(10,15)$

$\gamma_{u_{\text {com }}}=\operatorname{Diag}(15,15) \quad, \quad \gamma_{u_{r}}=\operatorname{Diag}(15,15)$

$\gamma_{\hat{v}_{i}^{\prime}}=\operatorname{Diag}(15,15)$.

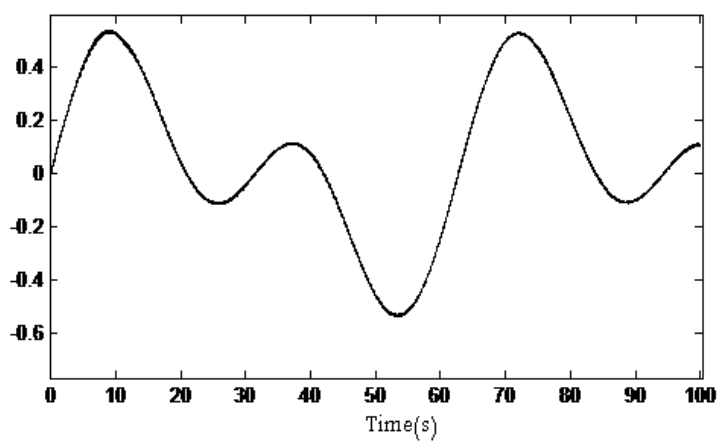

Fig. 2: the time trajectory of first state (dotted line) and the desire trajectory(solid line)

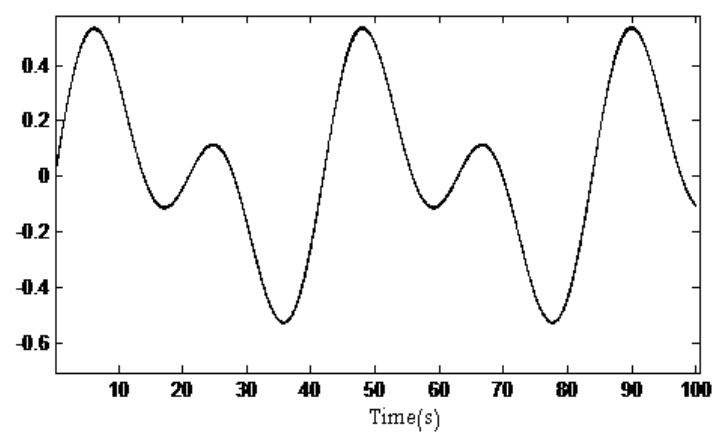

Fig. 3: the time trajectory of second state (dotted line) and the desire trajectory(solid line)

Fig. 2 and 3 show promising performance of the controller. The controller input are shown in the following figures.

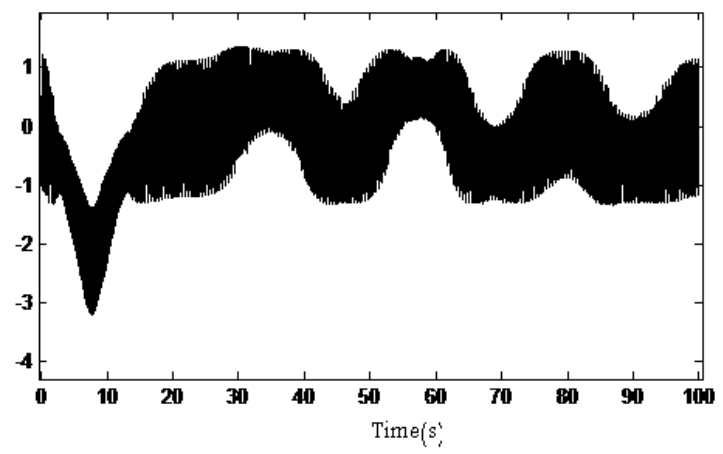

Fig.4: the first control input

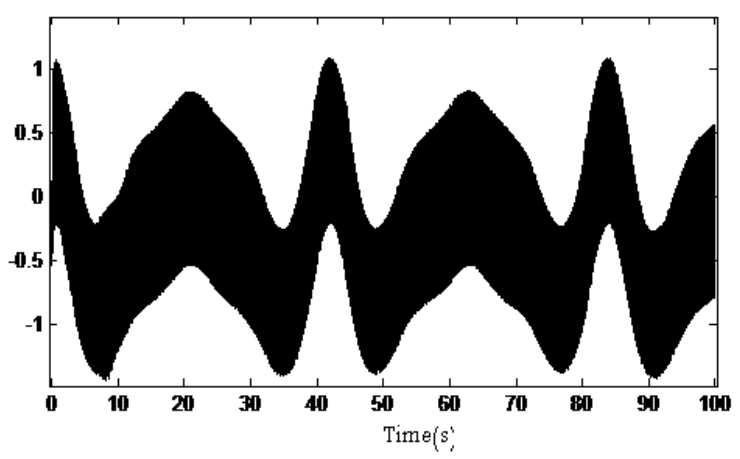

Fig. 5: the second control input

To show boundedness of the controller parameters, the following figures are proposed.

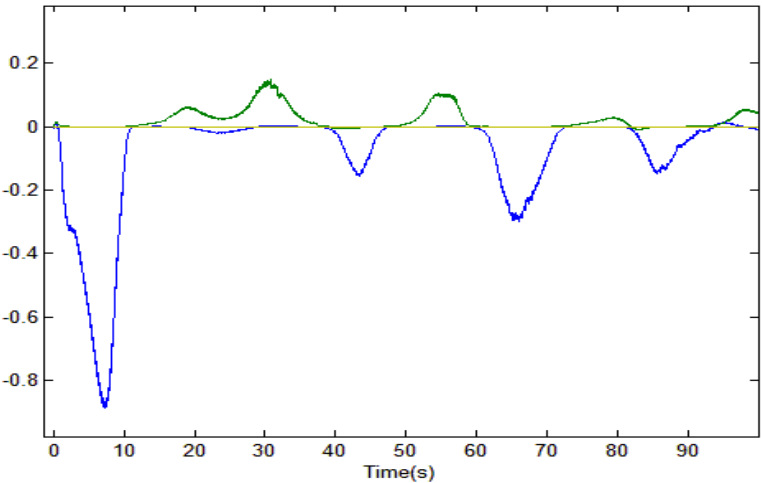

Fig. 6: parameters of fuzzy systems in first control input

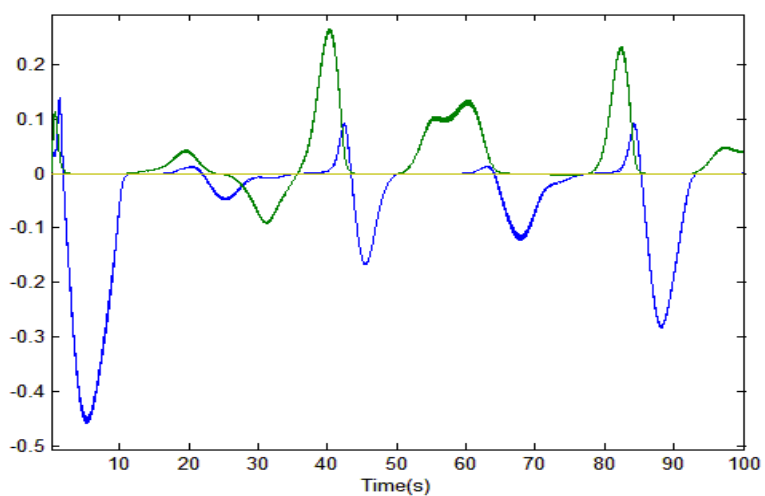

Fig. 7: parameters of fuzzy systems in second control input

\section{CONCLUSION}

In this paper, we propose a new model reference adaptive controller for a class MIMO non-affine nonlinear system. To apply knowledge experts in controller, Fuzzy systems are powerful tool in the controller design procedure. It has been shown that the derived adaptation laws guaranty the stability of closed-loop system, and asymptotic convergence of the tracking error to zero. Robustness against external disturbances and approximation errors, relaxing the conditions and using knowledge of experts are the merits of the proposed controller.

\section{REFERENCES}

[1] P. Ying-guo, Z. Hua-guang,'Design of fuzzy direct adaptive controller and stability analysis for a class of nonlinear system", Proceedings of the American Control conference, Philadelphia, Pennsylvania,pp. 2274-2275, June, 1998.

[2] W.S. Yu, "Model Reference Fuzzy Adaptive Control for Uncertain Dynamical Systems with Time Delays", IEEE International Conference on Systems, Man and Cybernetics, pp. 5246-5251,2004.

[3] H. J. Zimmermann, "Fuzzy set theory- and its application", Kluwer Academic publishers, 1996

[4] T. Yiqian, W. Jianhui, G. Shusheng, Q. Fengying, "Fuzzy Adaptive Output Feedback Control for Nonlinear MIMO Systems Based On Observe", Proceedings of the $5^{\text {th }}$ World Congress on Intelligent Control and Automation Hangzhou, P.R. China, pp. 506-510June 15-19, 2004.

[5] P. Ioannou, J. Sun, Robust Adaptive Control, Prentice-Hall, Inc., Englewood Cliffs, NJ, 1996, Electronic copy at <http://www-rcf.usc.edu/ioannou/Robust_Adaptive_Control.htm.

[6] C.C.Chiang, "Adaptive Fuzzy Sliding Mode Control For Time-Delay Uncertain Large-Scale Systems", Proceedings of the 44th IEEE Conference on Decision and Control, and the European Control Conference, pp. 4077-4082,Seville, Spain, December 12-15, 2005.

[7] [7] L.X. Wang, J.M. Mendel, "Fuzzy basis function, universal approximation, and orthogonal least square learning", IEEE Trans. Neural Network 3 -5 ,pp.: 807-814, 1993.

[8] L.X. Wang," A course in fuzzy systems and control", Prentice Hall PTR, 1997

[9] H. Khalil," Nonlinear Systems", prentice Hall, second Edition,1996. 
[10] H. Wu, "Decentralized Adaptive Robust Control for a Class of Large-Scale Systems Including Delayed State Perturbations in the Interconnections", IEEE TRANSACTIONS ON AUTOMATIC CONTROL, VOL. 47, NO. 10, pp.1745-1751, OCTOBER 2002.

[11] T. Shaocheng, C. Bin,W.Yongfu, "Fuzzy adaptive output feedback control for MIMO nonlinear systems", Elsevier Science, Fuzzy Sets and Systems 156, pp. 285-299, 2005.

[12] S.Tong, H.X. Li, W. Wang, "Observer-based adaptive fuzzy control for SISO nonlinear systems", Elsevier Science, Fuzzy Sets and Systems 148, pp. 355-376, 2004.

[13] H.F. Ho, Y.K. Wong , A.B. Rad, W.L. Lo, "State observer based indirect adaptive fuzzy tracking control", Simulation Modeling Practice and Theory 13, pp. 646-663, 2005.

[14] L. Zhang, "Stable Fuzzy Adaptive Control Based on Optimal Fuzzy Reasoning", IEEE, Proceedings of the Sixth International Conference on Intelligent Systems Design and Applications (ISDA'06), 2006.

[15] G. Fenga, S.G. Caoa, N.W. Reesb, "Stable adaptive control of fuzzy dynamic systems", Elsevier Science, Fuzzy Sets and Systems 131, pp. $217-224,2002$.

[16] G. Feng, "An Approach to Adaptive Control of Fuzzy Dynamic Systems", IEEE TRANSACTIONS ON FUZZY SYSTEMS, vol. 10, No. 2, pp. 268-275, APRIL 2002.

[17] Y.C. Hsu, G. Chen, S. Tong, H.X. Li, "Integrated fuzzy modeling and adaptive control for nonlinear systems", Elsevier Science, Information Sciences 153, pp.217-236, 2003.

[18] N. Goleaa, A. Goleaa, K. Benmahammed, "Stable indirect fuzzy adaptive control", Elsevier Science, Fuzzy Sets and Systems 137, pp. 353-366, 2003.

[19] C.W. Park, M. Park, "Adaptive parameter estimator based on T-S fuzzy models and its applications to indirect adaptive fuzzy control design", Elsevier Science, Information Sciences 159, pp. 125-139, 2004.

[20] C.C. Cheng, S.H. Chien, "Adaptive sliding mode controller design based on T-S fuzzy system models", Elsevier Science, Automatica 42, pp.1005-1010, 2006

[21] X. Jianga,W. Xua, Q.L. Hanb, "Observer-based fuzzy control design with adaptation to delay parameter for time-delay systems", Elsevier Science, Fuzzy Sets and Systems 152, pp. 637-649, 2005.

[22] S. Jagannathan, "Adaptive Fuzzy Logic Control of Feedback Linearization Discrete Time Dynamical Systems Under Persistence of Exitation", Automatica, vol. 34, no. 11, pp. 1295-1310, 1998.
[23] Y. Tang, N. Zhang, Y. Li, "Stable fuzzy adaptive control for a class of nonlinear systems", Elsevier Science, Fuzzy Sets and Systems 104,pp. 279-288,1999.

[24] L. Chen, G. Chen, Y.W. Lee, "Fuzzy modeling and adaptive control of uncertain chaotic systems", Elsevier Science, Information Sciences 121,pp. 27-37, 1999 .

[25] S. C. Tong, Q. Li, T. Chai, "Fuzzy adaptive control for a class of nonlinear systems", Elsevier Science,Fuzzy Sets and Systems 101, pp. 31-39,1999.

[26] S. Labioda, M. S. Boucheritb, T. M. Guerrac, "Adaptive fuzzy control of a class of MIMO nonlinear systems", Elsevier Science, Fuzzy Sets and Systems 151, pp.59-77, 2005.

[27] S. Labiod, T. M. Guerra, "Adaptive fuzzy control of a class of SISO nonaffine nonlinear systems", Elsevier Science, Fuzzy Sets and Systems 158, pp. 1126-1137, 2007.

[28] Y.J. Liu, W. Wang, "Adaptive fuzzy control for a class of uncertain nonaffine nonlinear systems", Elsevier Science, Information Sciences pp. 1-17, 2007.

[29] R. Ghasemi, M.B. Menhaj, A. Afshar, "A decentralized stable fuzzy adaptive controller for large scale nonlinear systems", Journal of Applied Science 9 (5), pp. 892-900, 2009.

[30] R. Ghasemi, M.B. Menhaj and A. Afshar, "A New Decentralized Fuzzy Model Reference Adaptive Controller for a Class of Large-scale Non-affine Nonlinear Systems" European Journal of Control ,Vol. 15, No. 5, p.p: 534-545, 2009.

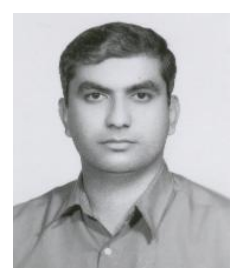

Reza Ghasemi (M'09) was born in Tehran, Iran in 1979. He received his B.Sc degrees in Electrical engineering from Semnan University in 2000 and M.Sc. degrees and Ph.D. in control engineering from Amirkabir University of Technology, Tehran, Iran, in 2004 and 2009.His research interests include large-Scale Systems, Adaptive Control, Robust Control, Nonlinear Control, and Intelligent Systems.

Dr. Reza Ghasemi joined Islamic Azad University, Damavand Branch, the Department of Electrical Engineering, Damavand, Tehran, Iran, where he is currently an Assistant Professor of electrical engineering. 